\title{
CASIMIR INTERACTION BETWEEN ABSORBING AND META MATERIALS
}

\author{
FRANCESCO INTRAVAIA $*$ and CARSTEN HENKEL \\ Institut für Physik, Universität Potsdam, 14469 Potsdam, Germany \\ francesco.intravaia@physik.uni-potsdam.de
}

\begin{abstract}
We investigate the Casimir energy between two dissipative mirrors in term of a sum over mode formula which can be interpreted by analogy to a quantum dissipative oscillator. We also show that metamaterials engineered at scales between the nanometer and the micron seem a promising way to achieve a repulsive force.

Keywords: Casimir effect; surface plasmon; dissipative materials; meta material; negative index.
\end{abstract}

The Casimir force is one of the most accessible experimental consequences of vacuum fluctuations in the macroscopic world. It is the most significant force between neutral, non-magnetic objects at distances on the micrometer scale and below. For many experiments searching for novel short-range forces predicted by unification models,${ }^{1-3}$ theoretical calculations of the Casimir force are crucial and have to be done at the same level of precision as the experiments. ${ }^{4}$

In this context, it is essential to account for the differences between the ideal Casimir case and real-world experiments, for example non-perfect reflectors made from absorbing material. This problem, in the plate-plate geometry, was solved by Lifshitz ${ }^{5}$

$$
F(L)=2 \hbar \operatorname{Im} \int_{0}^{\infty} \frac{\mathrm{d} \omega}{2 \pi} \operatorname{coth}\left(\frac{1}{2} \beta \omega\right) \int_{0}^{\infty} \frac{\mathrm{d} k k k_{z}}{2 \pi} \sum_{\lambda=\mathrm{TE}, \mathrm{TM}}\left(\frac{\mathrm{e}^{2 \mathrm{i} k_{z} L}}{r_{\lambda, 1} r_{\lambda, 2}}-1\right)^{-1},
$$

where $L$ is the distance between the plates, $\beta=\hbar / k_{B} T, k_{z}=\left(\omega^{2} / c^{2}-k^{2}\right)^{1 / 2}$, and $r_{\mathrm{TE}, \alpha}, r_{\mathrm{TM}, \alpha}$ are the reflection coefficients at plate $\alpha=1,2$ for the two principal polarizations of the electromagnetic field. This formula allows to calculate the Casimir force (per unit area) in terms of the optical properties of the plates, with any non-ideal behaviour (finite permittivity, dissipation) taken into account by suitable models for the reflection coefficients. For example, with a dissipative medium one uses a complex dielectric function provided it is compatible with causality constraints. ${ }^{6,7}$

Lifshitz' approach rather differs from the one used by Casimir. In fact, Casimir summed the zero-point energies of the electromagnetic modes inside a cavity of perfectly reflecting mirrors (Dirichlet boundary conditions), renormalizing this sum by removing the free vacuum energy. The Casimir energy for a cavity with real mirrors can also be obtained in this way, the modes being here the ones of the real cavity. ${ }^{8}$ Adopting a dissipation-less model for the dielectric function, the final expression coincides with Lifshitz' formula (10). Lifshitz theory has, however, a wider

*Supported by QUDEDIS (ESF program) and FASTNet (European Research Training Network). 
range of applicability because dissipative mirrors can also be described. We have shown that, also in this case, the Casimir effect can be expressed as a sum over modes. ${ }^{9}$ A calculation along lines similar to Ref.10 allows to transform Eq.(1) into (zero temperature, identical mirrors)

$$
F(L)=\frac{\partial}{\partial L} \frac{\hbar}{2} \int_{0}^{\infty} \frac{\mathrm{d} k k}{2 \pi} \operatorname{Re}\left[\sum_{n \lambda} \omega_{n \lambda}(k)-2 \mathrm{i} \frac{\omega_{n \lambda}(k)}{\pi} \log \frac{\omega_{n \lambda}(k)}{\omega_{c}}\right]_{L \rightarrow \infty}^{L},
$$

where $\omega_{c}$ is an arbitrary cutoff frequency and the discrete index $n$ labels the different modes that exist for a given $k$-vector and polarization. The frequencies $\omega_{n \lambda}(k)$ are the complex solutions of $\mathrm{e}^{2 \mathrm{i} k_{z} L} / r_{\lambda}^{2}-1=0$. Their imaginary parts obey a specific sum rule that removes the dependence on the cutoff $\omega_{c}$. The result (2) can be understood by analogy to the quantized oscillator coupled to a bath, establishing a bridge between the quantum field theory and the theory of open systems. At zero temperature, the oscillator's zero-point energy is shifted because the bath quantum fluctuations couple to the oscillator observables. ${ }^{11}$ The logarithmic term arises because the ground state of the uncoupled oscillator is no longer an eigenstate for the whole system, therefore its energy shows fluctuations.

In the non-dissipative case and at short distance, it is well known that the Casimir energy can be understood from the interaction between surface plasmon resonances on the two (metallic) mirrors. ${ }^{12}$ This holds also in the dissipative case. Adopting the lossy Drude model $\left(\varepsilon=1-\omega_{p}^{2} /\left(\omega^{2}+\mathrm{i} \gamma \omega\right)\right)$, one can show that Eq.(2) reduces to

$$
F(L) \approx\left(\frac{\alpha \omega_{p}}{2 \pi}-\frac{15 \zeta(3) \gamma}{\pi^{4}}\right) \frac{\hbar \pi^{2}}{240 L^{3}} \quad(\alpha=1.193 \ldots)
$$

where we have taken the leading order correction in $\gamma$ of Eq.(2) and kept in the sum only two modes, $\omega_{ \pm}=\frac{1}{\sqrt{2}} \sqrt{\omega_{p}^{2}\left(1 \pm e^{-k L}\right)-\gamma^{2} / 2}-\mathrm{i} \gamma / 2$, which are the dissipative counterparts of the coupled surface plasmons.

Lifshitz theory also allows to consider materials with engineered properties. Natural materials have a magnetic permeability which actually can be set always equal to one in the range of frequencies relevant for the Casimir effect. Nothing forbids, however, to consider artificial materials (also called metamaterials) which show a strong modification of their magnetic properties, say, in the visible-light range. We have recently investigated the simple case of a local magneto-dielectric material where both permittivity and permeability are given by lossy Drude models. ${ }^{13}$ More precisely, the permeability is

$$
\mu(\omega)=1+\frac{f \omega^{2}}{\omega_{0}^{2}-\omega^{2}-\mathrm{i} \kappa \omega}, \quad 0<f<1 .
$$

Response functions of this kind have been used previously to describe the response of a metamaterial to electromagnetic waves. The material contains a regular lattice of sub-wavelength units (wires and rings) with a size much smaller than the incident wavelength and filling factor $f$. With a suitable spatial averaging procedure (effective medium description), ${ }^{14}$ one finds the permeability (4). 
The calculation of the Casimir force requires response functions at imaginary frequencies. We have used the Kramers-Kronig relation

$$
\mu(\mathrm{i} \xi)=1+\frac{2}{\pi} \int_{0}^{\infty} \mathrm{d} \omega \frac{\omega \operatorname{Im} \mu(\omega)}{\omega^{2}+\xi^{2}}
$$

and focused on the limit of weak absorption where $\operatorname{Im} \mu(\omega)$ collapses to a $\delta$-function. The resulting expression features a "magnetic plasma frequency" $\omega_{p}=\omega_{0} \sqrt{f}$.

As shown in Fig 1, the Casimir interaction becomes repulsive for a 'mixed' pair of mirrors, one mainly dielectric, the other mainly permeable. This previously discussed phenomenon ${ }^{15}$ survives in some range of distances at sufficiently low temperatures even for dispersive materials. The corresponding parameter window is the wider, the higher the magnetic plasma frequency.

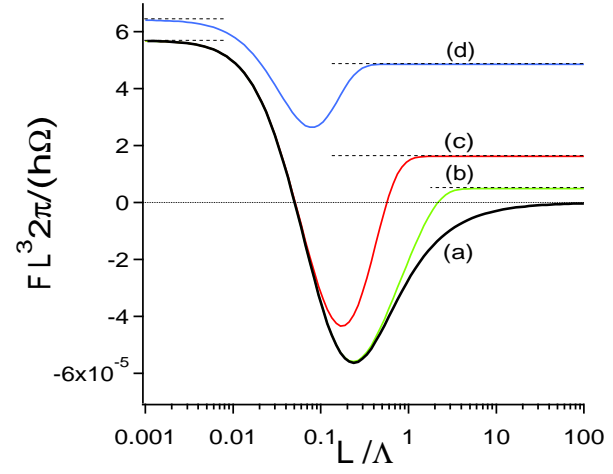

Fig. 1. Casimir pressure as a function of distance $L$ between two different metamaterial plates. Positive values correspond to an attractive interaction. The force (per unit area) is normalized to $\hbar \Omega / L^{3}$, the distance to $\Lambda \equiv 2 \pi c / \Omega$ where $\Omega$ is a typical plasma or resonance frequency in Eq.(4). Plate 1 is purely dielectric, plate 2 mainly magnetic. The temperature takes the values $k_{B} T=$ (a) 0, (b) 0.03, (c) 0.1, (d) $0.3 \hbar \Omega$. Adapted from Fig. 2 of Ref. 13 where the parameters can be found.

\section{References}

1. M. Bordag, U. Mohideen and V. Mostepanenko, Phys. Rep. 353, 1 (2001).

2. S. K. Lamoreaux, Rep. Progr. Phys. 68, 20 (2005).

3. R. Onofrio, New J. Phys. 8, 237 (2006).

4. A. Lambrecht and S. Reynaud, Eur. Phys. J. D8, 309 (2000).

5. E. Lifshitz, Sov. Phys. JETP 2, 73 (1956).

6. L. Landau, E. Lifshitz and L. Pitaevskii, Electrodynamics of Continuous Media (Pergamon Press, Oxford, 1980).

7. J. Jackson, Classical Electrodynamics (Wiley \& Sons, New York, 1975).

8. K. Schram, Phys. Lett. A43, 282 (1973).

9. F. Intravaia and C. Henkel, in preparation.

10. F. Intravaia and A. Lambrecht, Phys. Rev. Lett. 94, 110404 (2005).

11. K. E. Nagaev and M. Büttiker, Europhys. Lett. 58, 475 (2002).

12. N. G. V. Kampen, B. R. A. Nijboer and K. Schram, Phys. Lett. A26, 307 (1968). E. Gerlach, Phys. Rev. B4 (1971) 393; C. Henkel, K. Joulain, J.-P. Mulet and J.-J. Greffet, Phys. Rev. A69, 023808 (2004).

13. C. Henkel and K. Joulain, Europhys. Lett. 72, 929 (2005).

14. S. A. Ramakrishna, Rep. Prog. Phys. 68, 449 (2005).

15. T. H. Boyer, Phys. Rev. A9, 2078 (1974). 